\title{
Semiclassical treatment of symmetry breaking and bifurcations in a non-integrable potential
}

\author{
M V Koliesnik, Ya D Krivenko-Emetov, A G Magner* \\ Institute for Nuclear Research, 03680 Kyiv, Ukraine \\ and National Technical University of Ukraine, 03056 Kyiv \\ K Arita \\ Department of Physics, Nagoya Institute of Technology, Nagoya 466-8555, Japan \\ M Brack \\ Institute for Theoretical Physics, University of Regensburg, D-93040 Regensburg, Germany \\ PACS ReF: 03.65.Ge, 03.65.Sq, 05.45.Mt, 21.60.Cs
}

\begin{abstract}
We have derived an analytical trace formula for the level density of the Hénon-Heiles potential using the improved stationary phase method, based on extensions of Gutzwiller's semiclassical path integral approach. This trace formula has the correct limit to the standard Gutzwiller trace formula for the isolated periodic orbits far from all (critical) symmetry-breaking points. It continuously joins all critical points at which an enhancement of the semiclassical amplitudes occurs. We found a good agreement between the semiclassical and the quantum oscillating level densities for the gross shell structures and for the energy shell corrections, solving the symmetry breaking problem at small energies.

Keywords: Periodic orbit theory, Gutzwiller trace formula, symmetry breaking, level density and energy shell corrections.
\end{abstract}

\section{Introduction}

Semiclassical periodic orbit theory (POT) is a convenient tool for analytical studies of the shell structure in the single-particle level density of finite fermionic systems 1, 2, 3, 4, 5, 6. This theory relates the level density and energy shell corrections to the sum of periodic orbits and their stability characteristics, and thus, gives the analytical quantum-classical correspondence. Recent studies of the POT focused on overcoming catastrophe problems in the derivation of the semiclassical trace formulae arising in connection with symmetry breaking and bifurcation phenomena, where the standard stationary phase method (SPM) fails (see [1]). Semi-analytical uniform approximations solving these problems for the case of well separated pichfork bifurcations in the non-integrable HénonHeiles (HH) potential [7] were suggested in [8, 9, using the normal-form theory of non-linear dynamics [10, 11, 12, 13.

In the present work, we derive an analytical semiclassical trace formula for the level density of the $\mathrm{HH}$ potential, employing the improved stationary phase method (ISPM) [14] valid for arbitrarily dense sequences of pitchfork bifurcations near the saddle-point energy and for harmonicoscillator symmetry breaking in the limit of small energies. In this respect, the regular-to-chaotic transition in Fermi

\footnotetext{
*e-mail: magner@kinr.kiev.ua
}

systems becomes important for the understanding of its influence on shell correction amplitudes. Fig. 1] shows transparently such a transition through Poincaré Surfaces of Section (PSS) of the non-linear classical dynamics for the $\mathrm{HH}$ potential as a simple non-trivial example [5, 15, see also [16, 17, 18, for the PSS and Lyapunov exponents in the three-dimensional axially symmetric Legendre-polynomial and spheroidal billiards. As shown in this figure, the obvious transition from chaos to order occurs with decreasing energy $e$ of the particle from the saddle $(e=1)$ to a small energy (harmonic oscillator) limit $e \rightarrow 0$. We show below the relation of this behavior of the PSS to the amplitudes of oscillations in the level density (density of states) and total energy of fermion systems.

\section{Periodic-orbit theory in phase space}

The level density $g(E)$ is obtained from the semiclassical Green's function [1] by taking its trace in the phase-space Poincaré variables $Q, p$ [10, 11, 12, 13, 14]:

$$
\begin{aligned}
g_{\mathrm{sc}}(E)= & \frac{1}{(2 \pi \hbar)^{2}} \operatorname{Re} \sum_{\mathrm{ct}} \int \mathrm{d} Q \int \mathrm{d} p T_{y \mathrm{ct}}\left|\mathcal{J}_{\mathrm{ct}}(p, P)\right|^{1 / 2} \\
& \times \exp \left\{\frac{i}{\hbar}\left[\widehat{S}_{c t}(Q, p, E)-Q p\right]-\frac{i \pi}{2} \mu_{\mathrm{ct}}\right\} .
\end{aligned}
$$

Here $Q$ and $p$ are the final $x^{\prime \prime}$ and initial $p_{x}^{\prime}$ coordinates in the phase-space variables $x, y, p_{x}, p_{y}$ perpendicular to a reference classical trajectory (ct) in two dimensions, $T_{y \text { ct }}=m \oint d y / p_{y}$ is the primitive partial period of the $y$ motion along the ct, $\widehat{S}_{\mathrm{ct}}(Q, p, E)$ the generating function, $\mu_{\mathrm{ct}}$ the Maslov phase, and $\mathcal{J}_{\mathrm{ct}}(p, P)$ is the Jacobian for the transformation between the variables shown as its arguments. The ISPM generating function $\widehat{S}_{c t}(Q, p, E)$ is defined by

$$
\widehat{S}_{\mathrm{ct}}(Q, p, E)=S_{\mathrm{ct}}(Q, p, E)+q p,
$$

where $S_{\mathrm{ct}}(Q, p, E)$ is the action $S_{\mathrm{ct}}\left(\mathbf{r}^{\prime}, \mathbf{r}^{\prime \prime}, E\right)=$ $=\int_{\mathbf{r}^{\prime}}^{\mathbf{r}^{\prime \prime}} \mathbf{p} \cdot \mathrm{d} \mathbf{r}$ expressed in terms of the Poincaré variables 

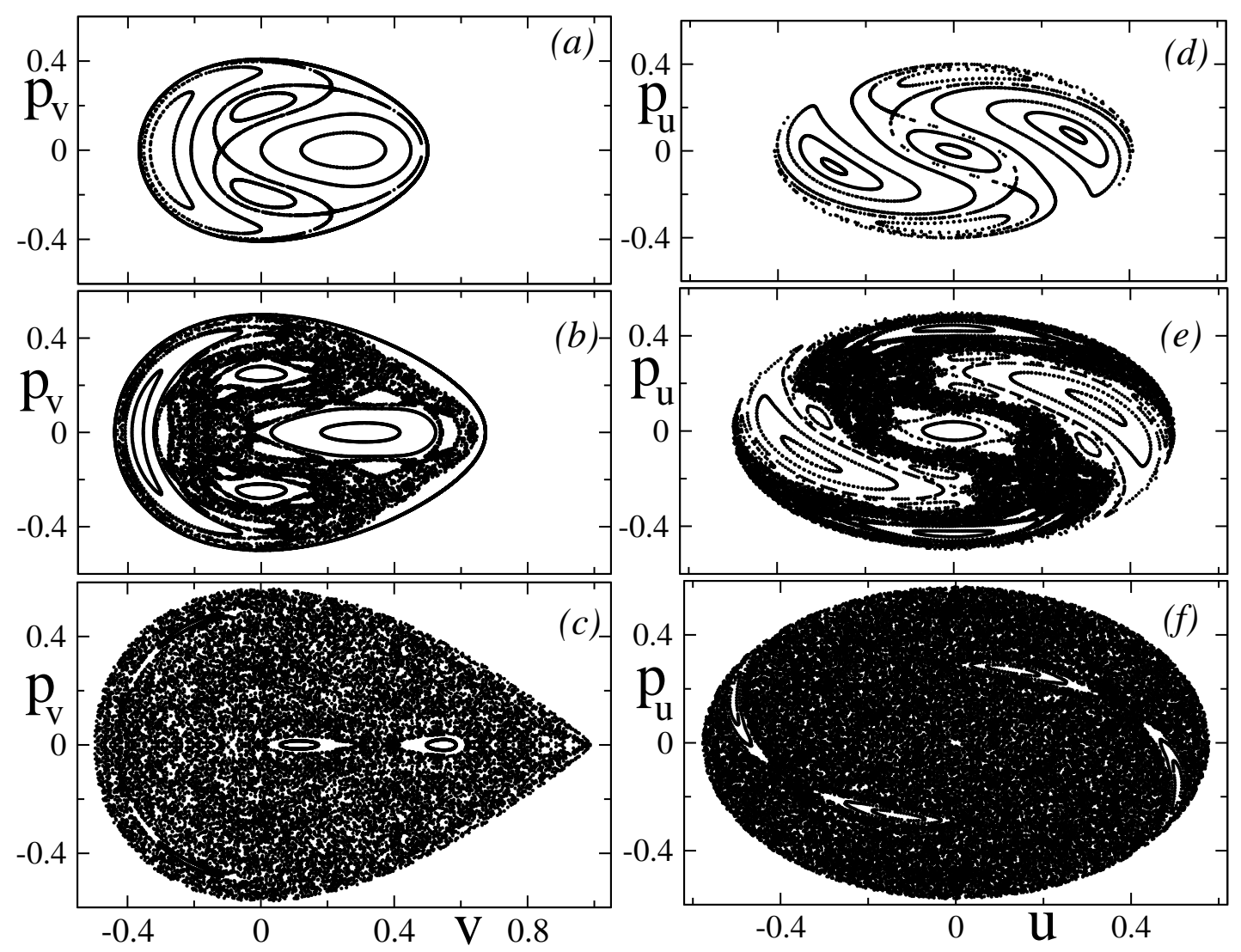

Fig. 1: Poincaré surfaces of sections (PSS) of the scaled Hénon-Heiles Hamiltonian $h$ (9); left column: (a), (b) and (c) plots show the PSS at $u=0$ for the energies $e=0.5,0.75$ and 1.0, respectively; right column: (d), (e) and $(f)$ graphics are given for $v=0$ at the same energies.

$Q$ and $p$ through the mapping transformation equations $Q=Q(q, p)$ and $P=P(q, p)$ along a ct $\left(\mathbf{r}^{\prime}\right.$ and $\mathbf{r}^{\prime \prime}$ are the initial and final spatial coordinates of the ct). It can be replaced by a (truncated) fourth-order expansion around the stationary points $Q^{*}, p^{*}$ which correspond to the periodic orbits (POs), $Q^{*}=q, p^{*}=P$ [14. For pichfork bifurcations, the expansion of the generating function $\widehat{S}_{\mathrm{ct}}(Q, p, E)$ (2) is similar to the normal forms [10, 11, 12, 13, with the following power series in $Q-Q^{*}$ and $p-p^{*}$ :

$$
\begin{aligned}
& S_{\mathrm{ct}}(Q, p, E)=S_{\mathrm{PO}}(E)+\epsilon_{\mathrm{PO}}^{(Q)}\left(Q-Q^{*}\right)^{2} \\
& \quad+a_{\mathrm{PO}}^{(Q)}\left(Q-Q^{*}\right)^{4}+\epsilon_{\mathrm{PO}}^{(p)}\left(p-p^{*}\right)^{2}+a_{\mathrm{PO}}^{(p)}\left(p-p^{*}\right)^{4},
\end{aligned}
$$

where $S_{\mathrm{PO}}(E)$ is the action along the PO. Performing also more exact integrations over $Q$ and $p$ in (1), one obtains for the case of pitchfork bifurcations

$$
\begin{aligned}
\delta g_{\mathrm{sc}}(E)= & \frac{1}{(2 \pi \hbar)^{2}} \operatorname{Re} \sum_{\mathrm{PO}} \frac{T_{\mathrm{PPO}}}{\left[\hbar^{2} a_{\mathrm{PO}}^{(Q)} a_{\mathrm{PO}}^{(p)}\right]^{1 / 4}} \mathcal{A}\left(\xi_{\mathrm{PO}}^{(Q)}\right) \\
& \times \mathcal{A}\left(\xi_{\mathrm{PO}}^{(p)}\right) \exp \left[\frac{i}{\hbar} S_{\mathrm{PO}}(E)-\frac{i \pi}{2} \sigma_{\mathrm{PO}}-i \phi\right],
\end{aligned}
$$

where $T_{\mathrm{PPO}}$ is the period for a primitive $\mathrm{PO}, S_{P O}(E)$ is its full action (including repetitions) at energy $E$, and

$$
\mathcal{A}(\xi)=\int_{z_{-}}^{z_{+}} \mathrm{d} z \exp \left[i\left(\xi z^{2}+z^{4}\right)\right], \quad \xi=\frac{\varepsilon}{(\hbar a)^{1 / 2}},
$$

is the amplitude factor; $\varepsilon$ and $a$ (for $a>0$ ) are the coefficients in the power expansion of the generating function $\widehat{S}_{c t}(Q, p, E)$ (2) with (3) in $Q-Q^{*}$ and $p-p^{*}$, which are proportional to the 2 nd and 4 th derivatives of $\left.\widehat{S}_{c t}(Q, p, E)\right)$ at the stationary points $Q^{*}$ and $p^{*} ; \sigma_{\mathrm{PO}}$ is the Maslov index related to the turning and caustic points along the POs, $\phi$ a constant phase independent of the PO. The integration (5) is performed over the finite classically accessible region of the Poincaré variables $Q$ and $p$, denoted here as

$$
z=\frac{Q-Q^{*}}{\left(a^{(Q)} / \hbar\right)^{1 / 4}}, \quad z=\frac{p-p^{*}}{\left(a^{(p)} / \hbar\right)^{1 / 4}}
$$

i.e. from $z_{-}^{(Q)}$ to $z_{+}^{(Q)}$ and from $z_{-}^{(p)}$ to $z_{+}^{(p)}$, respectively, with

$$
z_{ \pm}^{(Q)}=\frac{Q_{ \pm}-Q^{*}}{\left(a^{(Q)} / \hbar\right)^{1 / 4}}, \quad z_{ \pm}^{(p)}=\frac{p_{ \pm}-p^{*}}{\left(a^{(Q)} / \hbar\right)^{1 / 4}} .
$$

In (41), the sum runs over the straight-line orbits $A_{\sigma}$, the rotational orbits $R_{\sigma}$, and the librational orbits $L_{\sigma}$ of the standard HH Hamiltonian [7] (here in units with $m=\omega=$ $\hbar=1)$ :

$$
H=\frac{1}{2}\left(\dot{x}^{2}+\dot{y}^{2}\right)+\frac{1}{2}\left(x^{2}+y^{2}\right)+\alpha\left(x^{2} y-\frac{y^{3}}{3}\right) .
$$



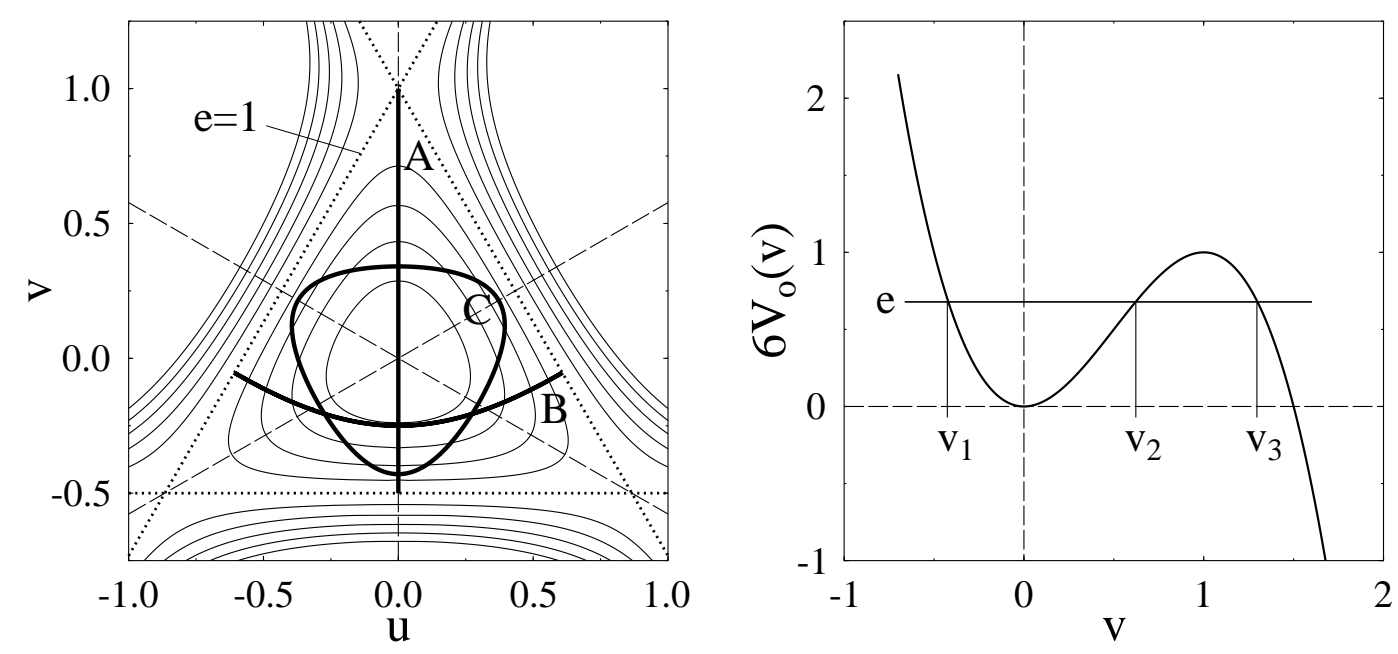

Fig. 2: The scaled Hénon-Heiles potential of the Hamiltonian (9). Left: Equipotential contour lines are given in scaled energies $e$ in the plane $(u, v)$. The dashed lines are the symmetry axes. The three shortest orbits $\mathrm{A}, \mathrm{B}$, and $\mathrm{C}$ (evaluated at the energy $e=1$ ) are shown by the heavy solid lines. Right: Cut along $u=0$ shows a barrier. (After [15].)

Using the barrier energy $E_{\text {barr }}=1 /\left(6 \alpha^{2}\right)$ as dimensionless energy unit, $e=E / E_{b a r r}=6 \alpha^{2} E$, and the following scaled variables

$$
\begin{aligned}
p_{u} & =\alpha p_{x}, \quad p_{v}=\alpha p_{y}, \quad u=\alpha x, \quad v=\alpha y, \\
h & =3\left(\dot{u}^{2}+\dot{v}^{2}\right)+3\left(u^{2}+v^{2}\right)+6 v u^{2}-2 v^{3},
\end{aligned}
$$

one obtains classical dynamic equations independent of the parameter $\alpha$

$$
\ddot{u}=-u-2 u v, \quad \ddot{v}=-v+v^{2}-u^{2} .
$$

The scaled HH potential is shown in Fig. 2 as equipotential lines, and the orbits $\mathrm{A}, \mathrm{B}$ and $\mathrm{C}$ (at $e=1$ ) are presented, too. The $\mathrm{HH}$ potential is invariant under rotations about 120 degrees, which leads to a discrete degeneracy of the orbits. Such a degeneracy can be simply taken into account multiplying the amplitudes in the trace formula (44) by a factor 3 . The cut along $u=0$ (right) shows a barrier at the saddle $e=1$ with two turning points $v_{1} \leq v_{2}$ at $0<e<1 ; v_{n}$ are the real solutions of the cubic equation $e=3 v^{2}-2 v^{3} \leq 1$ (A44).

In order to simplify the amplitude function $\mathcal{A}$ in the ISPM trace formula (4), we note that sufficiently far from the symmetry-breaking limit $E=0$, the integration limits in (5) can be extended to $\pm \infty$ (convergence being guaranteed by the finite fourth-order terms). Then, the amplitudes $\mathcal{A}$ (5) can be expressed through integral representations of the Bessel functions $J_{ \pm 1 / 4}(x)$ :

$$
\begin{aligned}
\mathcal{A}(\xi)= & \frac{\pi}{2} \sqrt{\xi}\left\{\exp \left[-i\left(\frac{\xi^{2}}{8}-\frac{\pi}{8}\right)\right] J_{-1 / 4}\left(\frac{\xi^{2}}{8}\right)\right. \\
& \left.-\frac{\xi}{|\xi|} \exp \left[-i\left(\xi+\frac{\pi}{8}\right)\right] J_{1 / 4}\left(\frac{\xi^{2}}{8}\right)\right\} .
\end{aligned}
$$

Here we took into account a time-reversal symmetry by inclusion of the factor 2 where necessary. Note that more exact trace formulae (with additional terms proportional to Bessel functions with indices $\pm 3 / 4$ etc.) can be derived by taking into account higher-order terms in the phase and amplitude factors, respectively. This gives results similar to those obtained in [11, 12, using the normal forms for pitchfork bifurcations.

Using asymptotic forms of the Bessel functions for large arguments $\xi$ in (11), one obtains from (4) (with $\phi=0$ ) the standard Gutzwiller trace formula [1, 5] valid for isolated POs:

$$
\begin{aligned}
& \delta g_{\mathrm{sc}}(E) \rightarrow \sum_{\mathrm{PO}} \mathcal{A}_{\mathrm{PO}}^{G}(E) \cos \left[\frac{S_{\mathrm{PO}}(E)}{\hbar}-\frac{i \pi}{2} \sigma_{\mathrm{PO}}\right], \\
& \mathcal{A}_{\mathrm{PO}}^{G}(E)=T_{\mathrm{PPO}}\left(\pi \hbar \sqrt{\left|2-\operatorname{Tr} \mathcal{M}_{\mathrm{PO}}\right|}\right)^{-1},
\end{aligned}
$$

where $\mathcal{M}_{\mathrm{PO}}$ is the stability matrix for the PO. Numerical and analytical calculations and the remarkable "fan" structure of the pitchfork bifurcations of the straight-line orbits $A_{\sigma}$ were analyzed in the case of the $\mathrm{HH}$ potential 20, 21, 22. As shown in the Appendix (Fig. 6), several analytical approximations for $\operatorname{Tr} M_{A}$ can be derived in terms of the simplest Mathieu functions for smaller energies $e \lesssim 0.8$, and in terms of improved Legendre solutions for the whole region from the zero energy to the saddle, $0 \leq e \leq 1$, in good agreement with the numerical results 20.

The trace formula (4) also has the correct harmonicoscillator $(\mathrm{HO})$ limit for $E \rightarrow 0$, where $\operatorname{Tr} \mathcal{M}_{\mathrm{PO}} \rightarrow 2$ and all coefficients in the expansion of the action phase in $Q$ and $p$ go to zero (and $\int d Q d p \rightarrow 2 \pi E$ ). The Poincaré variables $Q$ and $p$ become cyclic in this HO limit. In the spirit of the uniform approximations (see Sec. 6.3 of [5] and App. A of [9]) within the ISPM, we may use a canonical transformation from the variables $(Q, p)$ to new variables $(\widetilde{Q}, \widetilde{p})$ in which one has a simple analytical ex- 
pression for the $\mathrm{PO}$ amplitude $\mathcal{A}_{\mathrm{PO}}^{G}\left[1-\exp \left(-E / A_{\mathrm{PO}}^{G}\right)\right]$, instead of $A_{\mathrm{PO}}^{G}$ in (12), with the two correct limits to the HO trace formula [5] for $E \rightarrow 0$ and to the Gutzwiller trace formula (12) for large $E$. We should note that this procedure is not unique. Strictly mathematically, the reduction of the full $\widehat{S}_{\mathrm{ct}}(Q, p, E)(2)$ to any desired normal form by means of canonical transformations is generally not possible because, according to Arnold 23., "formal series for canonical transformations reducing a system to normal form generally diverge". On the other hand, within the ISPM, we use as "normal forms" equation (21) for the generating function with expansion (3) near the stationary points rather than near the bifurcations. A similarity to the normal form theory is manifested if we put formally $Q^{*}=0, p^{*}=0$ in (3) in the system of coordinates related to the bifucation point reducing the non-local ISPM to its local approximation valid nearly the bifurcation points. Moreover, from a more pragmatic point of view, the details of the required canonical transformation do not matter for the SPM approximation in narrow regions of phase space around the critical points, i.e., in the limit $\hbar \rightarrow 0$ which in practice corresponds to large particle numbers $N$ through the Fermi energy $E_{F}$ at a rather small parameter $\alpha$ and larger averaging width $\gamma$ of the gross shell structure. We emphasize also the chaos-to-order transition of the PSS in the limit to the symmetry breaking point $e \rightarrow 0$ (see also Fig. (1). In this limit, the isolated trajectories are transformed into the degenerate families of the periodic orbits.

Expressions found from (4) locally for the separate bifurcations of the $R$ or $L$ orbits are in agreement with the results [10, 11, 12, 13, obtained using the standard normal forms for the pitchfork bifurcations. However, for the full cascade of bifurcations near the saddle energy of the $\mathrm{HH}$ potential, our result (4) goes beyond the normal-form theory. It is a continuous function through all bifurcation points near the saddle energy and also down to the limit to the symmetry-breaking point at $E=0$. The coefficients $\varepsilon(E)$ and $a(E)$ in (5) are also continuous functions of the energy $E$ through all stationary points (POs). Note also that our ISPM expression (44) for the shell correction to the level density is a sum of separate contributions of all involved POs, and a coarse-graining over the energy $E$ (cf. below) may therefore be performed analytically. Thus, one has a possibility to study analytically both gross and fine shell structures. This is in contrast to the results [8, 9 ] using uniform approximations based on the normal-form theory [10, 11, 12, 13, where at each critical point all involved POs give one common contribution.

\section{Discussion of results}

Fig. 3 shows the modulus of the amplitude factor $|\mathcal{A}|$ (5) as function of $\xi=\varepsilon /(\hbar a)^{1 / 2}$. As seen from this figure, there is a typical enhancement of the amplitude $|\mathcal{A}|$ near the bifurcation point $\xi=0$. The ISPM amplitude factor (5D) has a finite value at the bifurcation point $\varepsilon=0$ and

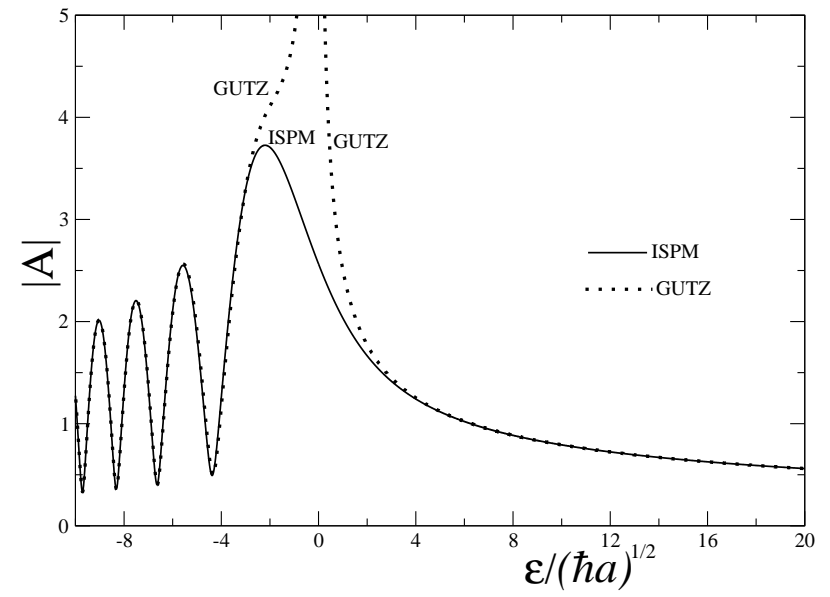

Fig. 3: The modulus of the amplitude factor $|\mathcal{A}|(5)$ as a function of $\varepsilon /(\hbar a)^{1 / 2}$ is plotted around the bifurcation point. The convergence of the ISPM to the Gutzwiller amplitude (GUTZ) far from the bifurcation is clearly seen.

converges to the Gutzwiller SSPM asymptotics $(\xi \gg 1)$ rather rapidly for $\xi>0$. As usual, one can see the characteristic "ghost" oscillations at negative $\xi$ which do not contribute into the semiclassical level density.

For the purpose of studying the improved level density around the bifurcation points, we consider a slightly averaged level density, thus avoiding the convergence problems that usually arise when one is interested in a full semiclassical quantization. Such a "coarse-graining" can be done by folding the level density over a Gaussian of width $\gamma$ [5, 6. (The particular choice of a Gaussian form of the averaging function is immaterial and guided only by mathematical simplicity.) Applying this procedure to the semiclassical level density (4), one gets [3, 5, 6]

$$
\delta g_{\gamma, s c}(E)=\sum_{\mathrm{PO}} \delta g_{\mathrm{sc}}^{(\mathrm{PO})}(E) e^{-\left(\frac{\gamma T_{\mathrm{PO}}}{\hbar}\right)^{2}}
$$

The averaging of the oscillating level density yields an exponential decrease of the amplitudes with increasing periods $T_{\mathrm{PO}}$ and/or $\gamma$. As shown in [14, for $\gamma$ about $1 / 3$ (in $\hbar \omega$ units), all large-action paths are strongly damped and only the time-shortest POs contribute to the oscillating part of the level density, yielding its gross-shell structure. For a study of the bifurcation phenomenon, however, we need smaller values of $\gamma$. In Figs. 4 and 5 we used the coarse-grained Gutzwiller trace formula (12]13) including the simplest primitive orbits $A, B=L_{4}$ and $C=R_{3}$.

It is interesting that the gross-shell structure manifests itself for the HH parameter $\alpha=0.04$ even for a relatively small averaging parameter $\gamma=0.25 \hbar \omega$. Therefore, we should expect also a good agreement between semiclassical and quantum results for the energy shell corrections $\delta U$ as functions of the particle numbers $N^{1 / 2}$ for the same $\alpha=0.04$ for larger energies (but still far enough from the bifurcation points, cf. Fig. (5).

The shell-correction energy $\delta U$, i.e., the oscillating part of the total energy $U$ of a system of $N$ fermions occupying 

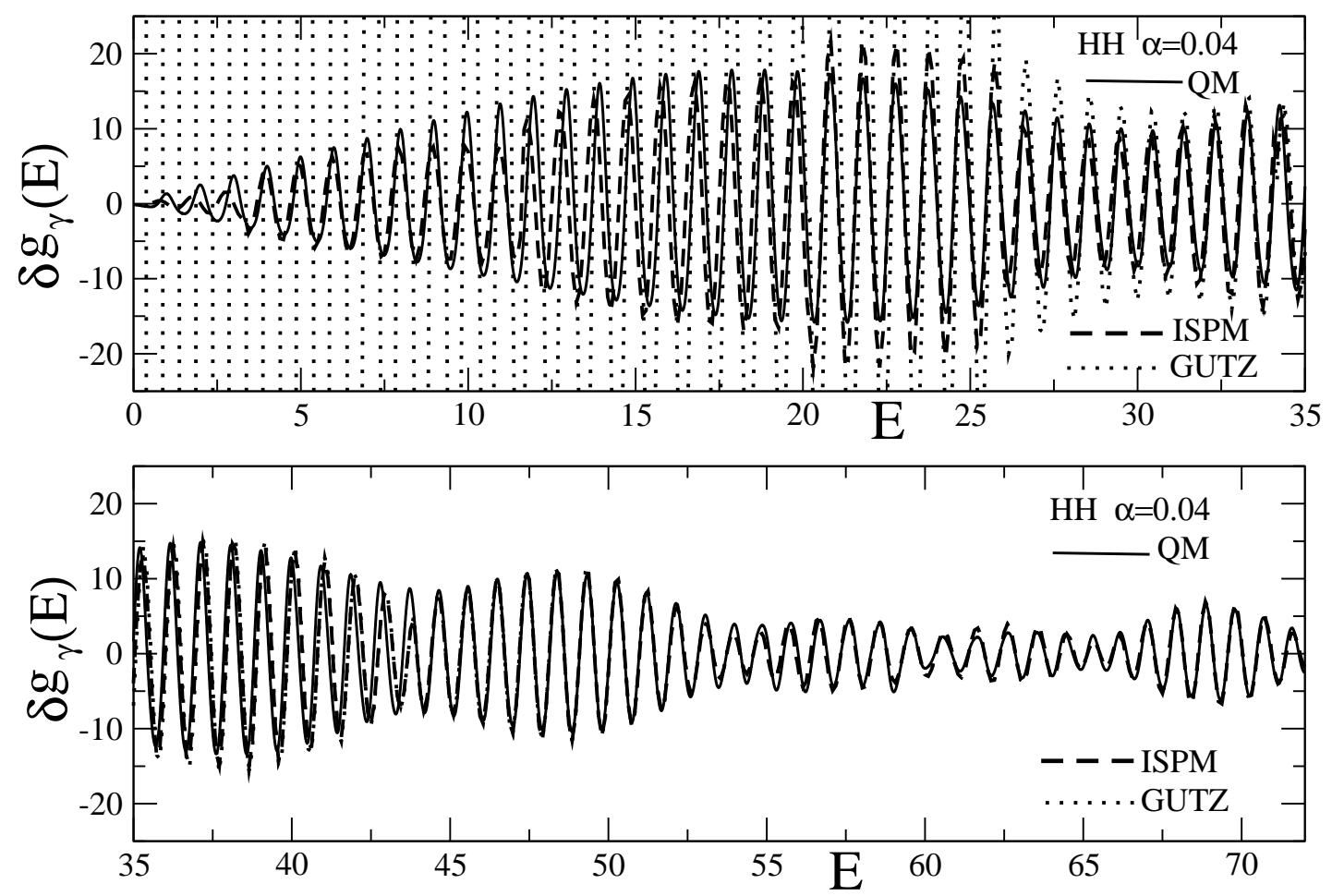

Fig. 4: Quantum (QM, solid), semiclassical (ISPM, dashed), and Gutzwiller (GUTZ, dots) level density versus energy E (in units of $\hbar \omega)$. Only the primitive POs A, B and C are included in the semiclassical calculations, the Gaussian averaging width is $\gamma=0.25 \hbar \omega$.
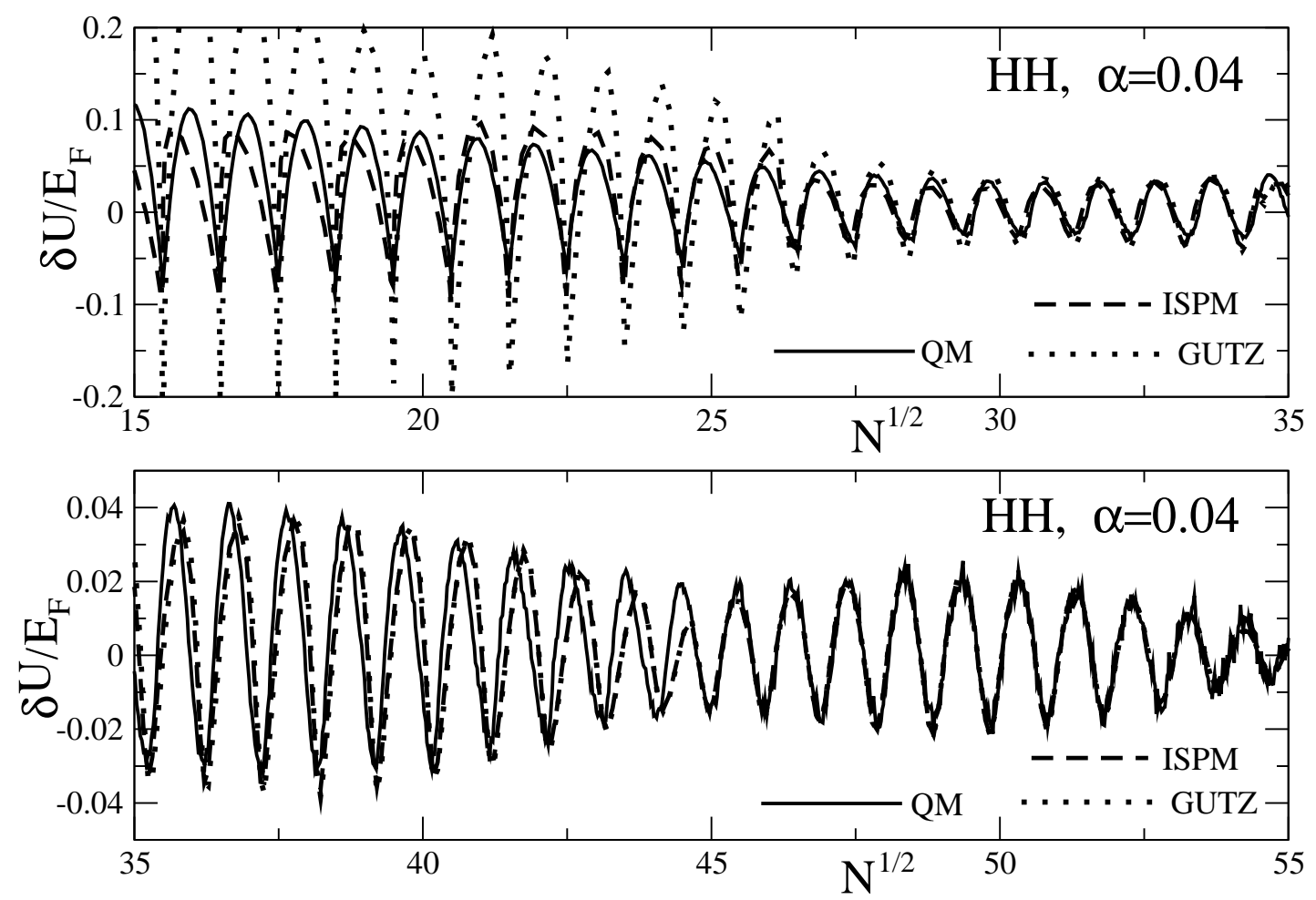

Fig. 5: Quantum and semiclassical energy shell corrections $\delta U$ (15) (in units of the Fermi energy $E_{F}$ ) versus particle number parameter $N^{1 / 2}$, with $N=2 \int_{0}^{E_{F}} d E g(E)$ (notation as in Fig. 4 ). The same primitive POs as in Fig. 4 are included. 
the lowest quantum levels in a given potential, can be expressed in terms of the oscillating components $\delta g_{\mathrm{sc}}^{\mathrm{PO}}(E)$ at the Fermi energy $E=E_{F}$ of the semiclassical level density,

$$
\delta g_{s c}(E)=\sum_{P O} \delta g_{s c}^{P O}(E)
$$

as [3, 4, 5, 6]

$$
\delta U=2 \sum_{\mathrm{PO}}\left(\frac{\hbar}{T_{\mathrm{PO}}}\right)^{2} \delta g_{\mathrm{sc}}^{\mathrm{PO}}\left(E_{F}\right) .
$$

Here, $T_{\mathrm{PO}}$ is the time of the motion along the PO including its repetitions at $E=E_{F}, T_{\mathrm{PO}}=M_{\mathrm{PO}} T_{\mathrm{PPO}}\left(M_{\mathrm{PO}}\right.$ is the repetition number). We are taking into account the spin degeneracy factor 2 in (15). The semiclassical representation of the shell-correction energy (15) differs from that of $\delta g_{s c}(E)$ (14) $\left(\right.$ at $\left.E=E_{F}\right)$ only by a factor $\left(\hbar / T_{\mathrm{PO}}\right)^{2}$ under the sum, which suppresses contributions from orbits of larger time periods (actions). Thus the periodic orbits with smaller periods play a dominant role in determining the shell-correction energy [3, 4]. Finally, we should note that the higher the degeneracy of an orbit, the larger the volume occupied by the orbit family in the phase space, and also the smaller its time period (action), the more important is its contribution to the level density.

Figs. 4 and 5 show a rather good agreement between the semiclassical and quantum results, in spite of using only the three shortest orbits $A, L_{4}(B)$, and $R_{3}(\mathrm{C})$. These are seen to yield the correct gross-shell structure for the parameter $\alpha=0.04$ and widths for the Gaussian averaging $\gamma=0.25 \hbar \omega$ (or, similarly, for $\gamma=0.6 \hbar \omega$ ) in the energy region below the saddle $\left(E=E_{\text {barr }}\right)$ and above the bottom $(E=0)$. The discrepancies at smaller energies are related to the symmetry breaking at $E=0$, as discussed above, and will be removed when using our full ISPM trace formula (4). In the quantum-mechanical determination of $\delta U$ (see Ch. 4.7 of [5] and [6, 14] for discussions of the Strutinsky averaging method), the plateau condition for the averaged energy was satisfied for a Gaussian width $\tilde{\gamma} \simeq 1.75 \hbar \omega$ and a curvature correction parameter $M=6$.

\section{Conclusions}

We have obtained an analytical trace formula for the oscillating part of the level density in Hénon-Helies Hamiltonian as a sum over periodic orbits. It is continuous through all critical points, in particular here the harmonic oscillator limit at zero energy and the cascade of pitchfork bifurcations near the saddle energy. We find an enhancement of the semiclassical amplitudes near the most critical points. The numerical agreement with quantum results is rather good, in spite of a rather simple uniform ISPM approximation including only the simplest primitive periodic orbits. The quantum-classical correspondence for the chaos-order transitions is shown through the Poincaré surface of sections in the limit from the non-integrable region of the energies to the symmetry breaking point. Our semiclassical analysis may therefore lead to a deeper understanding of the shell structure effects in finite fermionic systems such as nuclei, metal clusters or semiconductor quantum dots whose conductance and magnetic susceptibilities are significantly modified by shell effects (see [5, 6, 24, 25, 26] for examples).

\section{Acknowledgement}

We are grateful to K. Tanaka for many discussions and help with the numerical calculations. Some of the classical orbits and their properties were calculated using the program pos.for developed by Ch. Amann in 19. We also thank J.P. Blocki, S.N. Fedotkin and A.P. Kobushkin for many useful discussions. A.G.M. acknowledges the universities of Regensburg, Kyoto and Osaka (RCNP), the Nagoya Institute of Technology, the Japanese Society of Promotion of Sciences, and the National Centre of the Nuclear Research of Warsaw for very kind hospitality and financial support.

\section{Appendix A: The trace of the stability matrix for the A orbit}

For small energies $e$, the trace $\operatorname{Tr} \mathcal{M}_{A}$ can be expressed through Mathieu functions by using a general method of soving Hill's equation for the Poincaré coordinate $x(t)$ perpendicular to the A orbit directed along the $y$ axis (Fig. 2). The perturbation $x(t)$ (in scaled variables (9)) near the A orbit is determined by Hill's equation (10) for the HH Hamiltonian (9),

$$
\ddot{x}(t)+\left[1+2 y_{A}(t)\right] x(t)=0,
$$

where $y_{A}(t)$ is the periodic solution for the A orbit $[20,8$, 22 ,

$$
y_{A}=y_{1}+\left(y_{2}-y_{1}\right) \operatorname{sn}^{2}(z, k), \quad z=a_{k} t+F(\varphi, k),
$$

$\operatorname{sn}(z, k)$ is the Jacobi elliptic function [27] with argument $z$; its modulus $k$ and the constant $a_{k}$ are given by

$$
k=\sqrt{\frac{y_{2}-y_{1}}{y_{3}-y_{1}}}, \quad a_{k}=\sqrt{\frac{y_{3}-y_{1}}{6}} ;
$$

$y_{1}$ and $y_{2}$ are the lower and upper turning points,

$$
\begin{array}{ll}
y_{1}=\frac{1}{2}-\cos \left(\frac{\pi}{3}-\frac{\phi}{3}\right), & y_{2}=\frac{1}{2}-\cos \left(\frac{\pi}{3}+\frac{\phi}{3}\right), \\
y_{3}=\frac{1}{2}+\cos \left(\frac{\phi}{3}\right), & \cos \phi=1-2 e .
\end{array}
$$

$F(\varphi, k)$ is the incomplete elliptic integral of first kind as a function of $\varphi=\arcsin \left\{\left[\left(y_{0}-y_{1}\right) /\left(y_{2}-y_{1}\right)\right]^{1 / 2}\right\}, y_{0}=$ $y_{A}(t=0)$ is the initial value. Using the Fourier expansion of $\operatorname{sn}^{2}(z, k)$, one has 28

$$
\begin{aligned}
\operatorname{sn}^{2}(z, k)= & \frac{K(k)-E(k)}{k^{2} K(k)}-\frac{2 \pi^{2}}{k^{2} K^{2}(k)} \\
& \times \sum_{n=1}^{\infty} \frac{n s^{n}}{1-s^{2 n}} \cos \left(\frac{\pi n z}{K(k)}\right),
\end{aligned}
$$


where $s=\exp \left[-\pi K\left(\sqrt{1-k^{2}}\right) / K(k)\right]$ is Jacobi's Nome [27, $K(k)$ and $E(k)$ are the complete elliptic integrals of first and second kind, respectively.

For small energies $e$ where Jacobi's Nome $s$ is small, $s \rightarrow k^{2} / 16 \approx \sqrt{e} /(12 \sqrt{3})$ for $e \rightarrow 0(k \rightarrow 0$, see (A3) $)$, the convergence of the Fourier series (A5) is fast even for $e \lesssim 0.8(s \lesssim 0.04)$. For such energies, we may truncate the Fourier series approximately, keeping only the first $(n=1)$ harmonic term. After substitution of (A2) with the expansion (A5), a simple transformation of the time variable and the parameters in (A1) leads to the standard equation for the Mathieu equation [29]:

$$
\frac{\mathrm{d}^{2}}{\mathrm{~d} \tau^{2}} x(\tau)+[a-2 b \cos (2 \tau)] x(\tau)=0,
$$

with

$$
\begin{aligned}
\tau & =\pi z /[2 K(k)], \\
a & =\left(\frac{2 K}{\pi a_{k}}\right)^{2}\left\{1+2\left[y_{1}+\left(y_{2}-y_{1}\right) \frac{K-E}{k^{2} K}\right]\right\}, \\
b & =8 s\left(y_{2}-y_{1}\right) /\left[k^{2} a_{k}\left(1-s^{2}\right)\right] .
\end{aligned}
$$

The solution of this second-order ordinary differential equation can be sought as a linear superposition of the fundamental set of the even $M_{C}(a, b, \tau)$ and odd $M_{S}(a, b, \tau)$ Mathieu functions with arbitrary constants $C_{1}$ and $C_{2}$ :

$$
x(\tau)=C_{1} \mathrm{M}_{C}(a, b, \tau)+C_{2} \mathrm{M}_{S}(a, b, \tau) .
$$

Applying to (A8) the boundary conditions for calculations of the stability matrix elements $\mathcal{M}_{x x}$ and $\mathcal{M}_{\dot{x} \dot{x}}$ as in [22, one obtains the constants $C_{1}$ and $C_{2}$ and the following diagonal matrix elements:

$$
\begin{aligned}
& \mathcal{M}_{x x}=\left.\frac{x(T)}{x(0)}\right|_{\dot{x}(0) \rightarrow 0}=\frac{\mathrm{M}_{S, 0}^{\prime} \mathrm{M}_{C, T}-\mathrm{M}_{C, 0}^{\prime} \mathrm{M}_{S, T}}{\mathrm{M}_{C, 0} \mathrm{M}_{S, 0}^{\prime}-\mathrm{M}_{S, 0} \mathrm{M}_{C, 0}^{\prime}}, \\
& \mathcal{M}_{\dot{x} \dot{x}}=\left.\frac{\dot{x}(T)}{\dot{x}(0)}\right|_{x(0) \rightarrow 0}=\frac{\mathrm{M}_{S, T}^{\prime} \mathrm{M}_{C, 0}-\mathrm{M}_{C, T}^{\prime} \mathrm{M}_{S, 0}}{\mathrm{M}_{C, 0} \mathrm{M}_{S, 0}^{\prime}-\mathrm{M}_{S, 0} \mathrm{M}_{C, 0}^{\prime}},
\end{aligned}
$$

where primes means the partial derivatives of the Mathieu functions $M_{C}(a, b, \tau)$ and $M_{S}(a, b, \tau)$ with respect to $\tau$. The lower indices 0 and $T$ show the values at the initial $t=0$ and final $t=T$ times, and $T=T_{A}=2 K(k) / a_{k}$ is the period of motion of the particle along the A orbit. For the trace $\operatorname{Tr} \mathcal{M}_{A}$, one finally finds

$$
\operatorname{Tr} \mathcal{M}_{A}=\mathcal{M}_{x x}+\mathcal{M}_{\dot{x} \dot{x}}
$$

with the diagonal matrix elements given in A91.

For comparison, we recall the solution for the trace $\operatorname{Tr} \mathcal{M}_{A}$ near the saddle $e \rightarrow 1$ obtained in 21, 22 in terms of the Legendre functions by using in (A2) the approximation of the Jacobi elliptic function, $\operatorname{sn}(z, k) \approx \tanh (z)$, i.e., by the zero-order term of its expansion near the saddle in powers of $1-k^{2}$ [29]:

$$
\operatorname{sn}(z, k) \approx \tanh z\left[1+\frac{1}{4}\left(1-k^{2}\right)\left(1-\frac{z}{\sinh z \cosh z}\right)\right] .
$$

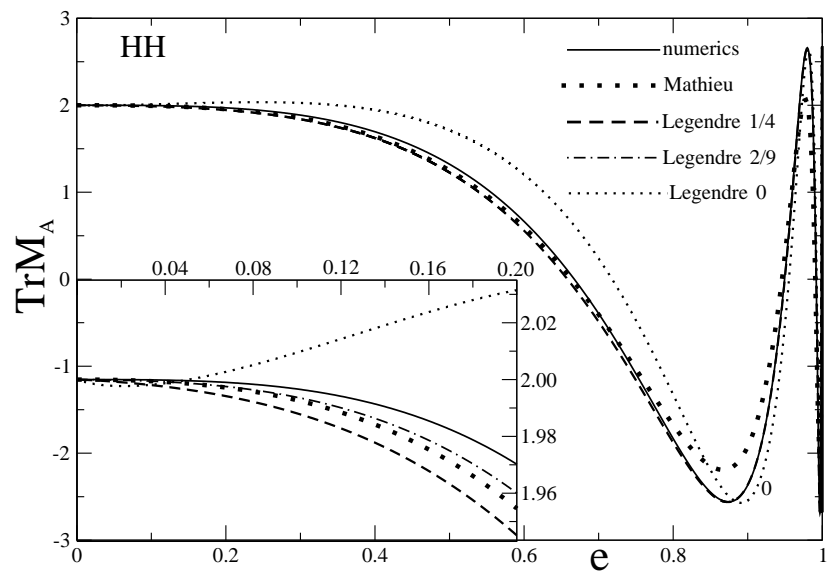

Fig. 6: Numerical and analytical traces $\operatorname{Tr} M_{A}$ for A orbit. The solid line is the full numerical result (after 20, 9]); heavy dots show the approximation (A10) through the Mathieu functions (A9); the dashed line shows the improved Legendre function approximation with the constant A12). Light dots present the asymptotic Legendre function approximation with $r=1$ $\left(r_{\text {corr }}=0\right)$. The insert shows a small region near the symmetry breaking limit $e \rightarrow 0$; the values of the constants $r_{\text {corr }}=0,1 / 4$ and 2/9 A12 for different Legendre function approximations are shown.

As shown in [21, 22], the trace $\operatorname{Tr} \mathcal{M}_{A}$ is in this approximation in good agreement with the numerical results 20 near the saddle $e \rightarrow 1$.

Generally speaking, for a more general solution, it is difficult to take into account exactly the next term of the expansion (A11) to get a simple analytical result similar to that presented explicitly in 22. However, we may use the approximate constant $r$ for the square brackets in (A11), which effectively takes into account the correction to $\tanh z$,

$$
r \approx 1+r_{\text {corr }}\left(1-k^{2}\right), \quad r_{\text {corr }}=1 / 4 .
$$

Within this approximation, one has again the result in terms of the Legendre functions $P_{\nu}^{\mu}$ and $Q_{\nu}^{\mu}$ with complex indices $\nu$ and $\mu$ depending on the energy $e$

$$
\mu=i \sqrt{A+B}, \quad \nu=(-1+i \sqrt{4 A-1}) / 2,
$$

where $B$ is the same as in 22 but $A$ contains the additional constant factor $r$ :

$$
A=12 r k^{2}, \quad B=\left(1+2 y_{1}\right) / a_{k}^{2},
$$

corresponding at $r=1$ (or $r_{\text {corr }}=0$ in our notations) to the results in 22]. Fig. [6 shows the comparison of numerical calculations 9, 20, with our analytical results for the trace of the stability matrix $\operatorname{Tr}_{A}$ in the case of the A orbit. As seen from Fig. 6. the solution for $\operatorname{Tr} \mathcal{M}_{A}$ in terms of the Mathieu functions is in good agreement with the exact numerical results even at energies $e \lesssim 0.8$. We show there also another approximation in terms of Legendre functions with the indices (A13), improved at finite and small energies $e$ through the constant $A$ (A14) with $r$ given in (A12) 
as compared to the result $(r=1)$ obtained earlier near the saddle [i.e., using only the leading term in the expansion (A11) for $e-1 \ll 1]$ [22. Through a modification of only one constant $r$ A12 , one has a remarkable agreement between this improved Legendre approximation and the numerical results everywhere from the saddle point $e$ to the harmonic oscillator limit $\operatorname{Tr} \mathcal{M}_{A} \rightarrow 2$ for $e \rightarrow 0$ (Fig. 6).

As shown in the insert of Figure 6 this approximation can be slightly improved changing the constant $r_{\text {corr }}$ in (A12) from $r_{c o r r}=1 / 4(z \rightarrow \infty)$ to about $2 / 9$ of finite values of $z$. For small energies $e(k \rightarrow 0)$, one can, again, formally use (A11): the correction to tanh $z$ can be neglected for small times $(z \ll 1)$ because it gives the dominating contribution to $\operatorname{Tr} M_{A}$ [equation (A1) with (A2) becomes approximately the same at small $z]$, and $\operatorname{Tr} M_{A} \rightarrow 2$ in all analytical approximations, in agreement with the numerical results. In the limit $e \rightarrow 0$ the Legendre function approximation converges, indeed, to the analytical Mathieu function solution (see the insert). Note also that this agreement with the numerical results is not sensitive to a variation of the constant $r_{\text {corr }}$ around the analytical value (A12). The particle moving near the A orbit spends much more time near the saddle where the function of $z \propto t$ in the circle brackets A11 is almost constant with respect to the remaining part of the trajectory. However, at small energies $e \rightarrow 0$, one finds a smaller time region $(z \ll 1)$ where the correction in A11 becomes negligible for $\operatorname{Tr} M_{A}$, such that all curves in Fig. 6 have the same correct harmonic oscillator limit 2. Thus, a rather complicated function of time in the correction to the leading (hyperbolic tangent) term of the expansion of the Jacobi function A11 can be reduced to a form involving the same Legendre functions as in [22, but with modified indices by the constant $r$ A12 through A14.

\section{References}

[1] Gutzwiller M 1990 Chaos in Classical and Quantum Mechanics (Springer-Verlag, New York)

[2] Strutinsky V M 1975 Nukleonika 20679

[3] Strutinsky V M and Magner A G 1976 Sov. Phys. Part. \& Nucl. 7138

[4] Strutinsky V M, Magner A G, Ofengenden S R and Døssing T 1977 Z. Phys. A 283269

[5] Brack M and Bhaduri R K 2003 Semiclassical Physics; Frontiers in Physics No 96, 2nd ed. (Westview Press, Boulder, CO)

[6] Magner A G, Yatsyshyn I S, Arita K and Brack M 2011 Phys. Atom. Nucl., 741445

[7] Hénon M and Heiles C 1964 Astr. J. 6973

[8] J. Kaidel and M. Brack 2006 Phys. Rev. E 70016206

[9] M. Brack and K. Tanaka 2008 Phys. Rev. E 77046205

[10] Sieber M 1997 J. Phys. A: Math. Gen. 304563.
[11] Schomerus H and Sieber M 1997 J. Phys. A: Math. Gen. 304537

[12] Sieber M and Schomerus H 1998 J. Phys. A: Math. Gen. 31165

[13] Schomerus H 1998 J. Phys. A: Math. Gen. 314167

[14] Magner A G, Fedotkin S N and Arita K 2006 Prog. Theor. Phys. 115523

[15] Brack M, Bhaduri R K, Law J and Murthy M V N 1993 Phys. Rev. Lett. 70568

[16] Blocki J P, Magner A G and Yatsyshyn I S 2010 At. Nucl. Energy 11239

[17] Blocki J P, Magner A G and Yatsyshyn I S 2011 Int. J. Mod. Phys. E 20, 292 (2011)

[18] Blocki J P and Magner A G 2012 Phys. Rev. C 85 064311

[19] Amann Ch and Brack M 2002 J. Phys. A: Math. Gen. 356009

[20] Brack M, Mehta M and Tanaka K 2001 J. Phys. A: Math. Gen. 348199

[21] Brack M, Kaidel J, Winkler P and Fedotkin S N 2006 Few-Body Syst. 38147

[22] Fedotkin S N, Magner A G and Brack M 2008 Phys. Rev. E 77066219

[23] Arnold V I 1989 Mathematical Methods of Classical Mechanics (New York: Springer-Verlag, 2nd edition), Addition 7

[24] Magner A G, Vlasenko A A and Arita K 2013 Phys. Rev. E 87062916

[25] Frauendorf S, Kolomietz V M, Magner A G and Sanzhur A I 1998 Phys. Rev. B 585622

[26] Magner A G, Gorpinchenko D V and Bartel J 2014 Phys. At. Nucl. 771229

[27] Byrd P F and Friedman M D 1971 Handbook of Elliptic Integrals for Engineers and Scientists (2nd ed, Sprinder-Verlag New York Heidelberg Berlin)

[28] Milne S C 2002 Infinite Families of Exact Sumes of Squares Formulas Jacobi Elliptic Functions, Continued Fractions, and Schur Functions (Kluwer Academic Publishers); the Ramanujan Journal 67

[29] Abramovitz M and Stegun I A 1965 Handbook of mathematical functions (Dover Publications, New York) 